\section{Rosacea and its Comorbidities}

\author{
Andreea Merticariu' ${ }^{1}$, Luminița Marinescu², Călin Giurcăneanu ${ }^{3,4}$
}

"St. Pantelimon" Clinical Emergency Hospital, Bucharest, Romania

${ }^{2} \mathrm{CMI}$ Marinescu Luminita, Bucharest, Romania

3" Elias" Clinical Emergency Hospital, Bucharest, Romania

"'Carol Davila" University of Medicine and Pharmacy, Bucharest, Romania

\section{ABSTRACT}

Objectives: Rosacea is currently defined as an inflammatory cutaneous disease without systemic echoes. The objective of our paper is to review the published articles to confirm our belief that rosacea is associated to or can predispose to various diseases, mainly inflammatory diseases.

Material and methods: Review all the papers published in the last two decades in PubMed, Google Academic and Medscape databases, linking rosacea and various disorders of the body systems.

Results: We found that rosacea is associated to several illnesses of major systems (gastrointestinal, cardiovascular, respiratory) and that there are also links between rosacea and immune system disorders and psychiatric diseases. Rosacea clinical course can be influenced by various drugs prescribed for the previous mentioned diseases.

Conclusions: Rosacea should no longer be considered a localized cutaneous condition but rather a complex pathology with systemic reverberation. Patients suffering of severe forms of rosacea for a long period of time should have a careful evaluation of body systems.

Key words: rosacea, association, link, inflammatory

\section{INTRODUCTION}

Rosacea (RS) is a chronic inflammatory recurrent facial disorder, frequently diagnosed in everyday practice. The patients' age at onset ranges between 30 and 35 years, but young and old people can also be affected. The medical literature mentions cases of facial dermatologic disorders in children with typical skin symptoms of RS. The disease is typically diagnosed in patients with sun sensitivity and skin phototype I and II, Northern or Eastern European origin, although all skin types can be affected. There is a predominance for female gender, but males generally have more severe clinical forms, including rhinophyma or gnathophyma. According to international grading systems, RS has four well established forms: erythematotelangiectatic, papulopustular, phymatous and ocular. Many clinicians also include in this category perioral dermatitis,
Corresponding author: Andreea Merticariu, MD "St. Pantelimon" Clinical Emergency Hospital, Bucharest, Romania E-mail: andreeamerticariu@yahoo.com 
corticoid induced RS, neurogenic RS, granulomatous RS, RS conglobata, and RS fulminans. These last forms are the most severe because they're also accompanied by high fever.

Patients describe in the first stages of the disease transient erythema spread on the centrofacial area or on the décolletage. In the course of the disease, under trigger factors influence telangiectasias on erythematous background, papulo-pustules and even sebaceous glands rich tissue hypertrophy (phymatous stage) will gradually develop. Chronic inflammation may lead to chronic edema and fibrous changes within the skin. There are many factors that can trigger RS flare ups and often prevention is hard to achieve. Several factors, including exposure to high or low temperature and significant sudden temperature changes, hot steam, direct sunlight, wind, spicy food or hot beverage consumption, alcohol (red wine), exercise, stress and unsuitable cosmetics or drugs applied to the sensitive skin of RS patients can lead to flushing that usually precedes or worsens the erythemato-telangiectatic and papulo-pustular stages. Despite that many therapeutic options are currently available and are able to induce obvious clinical improvement, none of them actually cures the disease, amplifying the anxious state of the patients.

Many etiopathogenic hypothesis are still being debated and there is no consensus on the etiopathogenetic mechanism of RS. Recent clinical studies indicate changes or anomalies of local vasculature, sebaceous glands, skin microbioma and/ or innate immune system. Modifications of the extracellular matrix which are specific to inflammation appear even from the early erythemato-telangiectatic phase, but inflammatory clinically signs are noticeable later in the course of this disease, respective when papules and/ or pustules develop.

\section{MATERIAL AND METHODS}

RS is currently considered a disease limited to the skin without any systemic influence. However, taking into account the well documented inflammatory phenomenon and the example of other inflammatory dermatologic diseases, whose systemic effects are acknowledged lately, we believe that RS could predispose or associate to other inflammatory diseases in its evolution. As a consequence, we initiated a thorough scientific literature search, looking for associations between RS and systemic diseases reported in various studies.

\section{RESULTS}

This way, we noticed that authors focused mainly on the connections between RS and gastro-intestinal affections. Even in the 1930's, doctors suspected a link of causality between RS and gastro-intestinal pathology. Etiopathogenic hypothesis from that period of time connected RS skin lesions development to gastric secretion disorders. One of the studies found hypochlorhydria in $75 \%$ of the patients involved in the study and in $12,5 \%$ hydrochloric acid secretion was near the inferior limit of the normal values interval. One other study from that time indicated reduced gastric secretion in about two thirds of the patients (1).

The most ample study dedicated to the RS - gastrointestinal system disorders association discovered the link between RS and Gram negative spiral bacteria Helicobacter Pylori (HP) which infects gastric mucosa. For a long time clinicians suspected that HP is directly implicated in RS etiopathogenesis, despite the fact that the mechanisms which lead to each specific lesion were not proven. Most researchers state that the proinflammatory background induced by HP gastric infection is responsible for the initialization of the cutaneous vascular and inflammatory changes that precede RS lesions.

Other authors suggest a causal relationship between the two disorders, based on the RS clinical improvement after HP treatment $(2,3,4,5)$ and the high incidence of this microorganism in RS patients $(6,7)$. Improvment in the symptomatology of the disease noticed after the treatment of HP infection is supported by several studies, while other authors consider that this treatment and RS evolution are not connected $(8,9)$. Some authors go even further and claim that RS is in fact an extragastric manifestation of HP infection $(2,10,11)$. However, there is still a lot of debate in the medical literature regarding whether or not HP has a high prevalence in RS patients. While some articles rule out this association (12-15), others confirm a statistic significant association HP - inflammatory papulopustular RS (16). Szlachcic et al. proved that RS is associated with a high virulent strain of HP. In their study, $67 \%$ of the patients with RS with dyspeptic symptoms had anti-CagA (cytotoxin associated gene A) antibodies compared to $32 \%$ of the patients without RS but complaining of dyspepsia (11). At a later date Argenziano et al. confirmed a predominance of anti-Cag A antibodies in RS patients (10) and El-Khalawany $M$ et al. recently proved that this virulent strain of HP is more frequently positive in papulo-pustular cases and its presence is responsible 
for an ampler inflammatory response in both gastric mucosa and cutaneous lesions (7).

It is yet not completely understood the precise mechanism in which HP infection of the gastric mucosa induces rosacea lesions. However, there is a consensus that cytokines and proinflammatory interleukins synthesized as a consequence of this infection, play a role in the initialization of cutaneous vascular and inflammatory changes that precede RS. Another etiopathological pathway could be high gastin secretion secondary to gastric infection, which leads to amplified cutaneous vascular reactivity and clinical transient flushing. Recently, Mary Ann Robledo and Mariana Orduz proposed a new hypothesis for flushing ignition and neoangiogenesis specific for RS, in HP positive patients. These authors believe that there is a defect of aldehid dehidrogenasis responsible for the late metabolism of high quantities of acetaldehid released secondary to HP infection, which ultimately leads to facial flushing (17).

Not just microorganisms resident in the stomach (HP) were suggested to cause RS, but also others located in the small intestine. Exaggerated overgrowth of the bacterial population from the small intestine was especially identified in the group of RS patients compared to control group in Coyle's study (18). Another study indicated RS symptoms amelioration on long term (over 9 months) after prescription of antibiotics without gastrointestinal absorption for patients diagnosed with overgrowth of the small intestine bacteria (19). The same authors later stated that there is a comparative prevalence of small intestine bacteria overgrowth in both patients and witness groups.

Regarding an association between other gastrointestinal pathologies and RS, studies published after the year 2000 indicate a high incidence of chronic active gastritis especially in the antrum and the stomach body in patients with RS versus sham $(2,11)$. The author's conclusion was that RS can frequently associate with antrum gastritis.

In medical literature there are published some RS fulminans cases associated with inflammatory bowel diseases. RS appears to be more frequently associated with ulcerative colitis than Crohn disease. Inflammatory bowel disease ethiopathology implies an interaction between human organism and intestinal bacteria, which leads to kinin-kalicrein system activation. The same system is activated in the skin of RS patients and this is why rosacea is thought to be caused by the neurogenic inflammatory syndrome induced by the above mentioned infectious agents (20). In 2015
Wen-Qing $\mathrm{Li}$ at al. conducted a retrospective study regarding the association between RS, use of tetracycline and risk of incident inflammatory bowel disease and concluded that RS patients have a minimal risk of ulcerative colitis and a high risk of Crohn disease, which increases with the duration of the disease. Also long term use of tetracycline correlates with an increased risk for both inflammatory bowel disease. The authors gathered their data from the Nurses' Health Study II (1991-2011) (21).

In 2004 Kendall reported a case with full RS remission in a patient who was administered/ treated with medical material which lowered intestinal transit period under 30 hours. The author also believes that facial flushing is a consequence of the neurogenic inflammation caused by the hyper sensibility of facial neurons, due to a kinin-kalikrein system activation under the influence of intestinal bacteria. Although this case sustains the hypothesis of rosacea- gastrointestinal conditions connection, additional studies regarding the length of the intestinal transit in RS patients should be conducted (22).

The possible connections between RS and inflammatory ENT diseases have also been studied. For instance, Al-Balbeesi proves in one study that there is a statistically significant association between $\mathrm{RS}$ and chronic rhinosinusitis. The patients with both diseases developed more severe forms of erythematothelangiectatic RS (23).

Recent studies suggest that there actually is a connection between peripheral nervous system and RS. In a lot of 140 patients with facial distonias (essential blefarospasm or hemifacial spasm), $15 \%$ of the patients were diagnosed with RS, compared to the 1,34\%, which is the prevalence of RS in the general population from which the group had been selected (24). The authors suggest the existence of some common immune- inflammatory pathways for both disorders as a possible explanation for this observation. Another article studied the RS - migraine association and it concluded that women older than 50 years suffering of severe migraine have a higher risk of developing RS. The same study indicated a slight increase of RS risk in persons older than 60 years who use Triptan, but this fact rather reflects the severity of the migrenous crisis than a real postmedicamentous effect (25). There are also citations in scientific journals of individual cases of unilateral RS, localized only on the hemiface affected by facial nerve palsy $(26,27)$.

Various authors searched a possible link that connects RS and the cardiovascular system. Brinnel $\mathrm{H}$ et al. proved on a small lot of patients that RS correlates 
with a deficient defense against brain overheating. Their study was designed based on the hypothesis that RS is a direct consequence of a deficiency in the venous drainage territory of the two facial veins and their main tributary veins, this vascular system being implicated in selective brain cooling. The authors concluded that their hypothesis is correct: the venous flow from the brain to the facial skin is suppressed under increase of cerebral temperature causing perturbation in the defense against cerebral overheating. The cause of these functional vascular anomalies could be in fact a defective contro exerted by the central nervous system on periphery (28). Vascular anomalies are also described in other vascular territories in patients suffering of RS. Hereby, in one study the capillary system of the nail bed was assessed with conventional capillaroscopy. All of the sixteen patients examined had anomalies of the microvasculature, where as only $63 \%$ of the control subjects displayed the same modifications, sustaining the authors' idea that conventional capillaroscopy investigation could be helpful in establishing the diagnosis of RS (29). Rosina P et al also used videocapillaroscopy to examine the facial and nail bed vascular configuration in patients diagnosed with either RS or seborrheic dermatitis and in healthy controls. This way there were identified specific vascular modifications in RS patients, as opposed to the other two groups: neoangiogenesis, proeminent telangiectasias and increased vascular diameters (30). On the other hand, in 2009, Fonseca GP et al could not detect on a small lot of patients, morphological modifications of the nail bed vessels that could be characteristic to patients suffering of erythematotelangiectatic RS in comparison to healthy controls. There for, the authors sustain that videocapillaroscopy as a diagnostic tool should be reevaluated in more comprehensive studies (31).

According to Duman $\mathrm{N}$ et al RS patients have a higher risk of cardiovascular diseases (32). The same conclusion was reached by Hua TC et al in 2015: RS patients have a higher risk to develop hypertension, dyslipidemia et coronary disease (33). Rainer BM et al. also stated in one poster that severe RS forms have a higher risk for essential hypertension (34). In 2015, Rainer BM et al. underlined that moderate to severe RS was more likely to be associated with hyperlipidemia, hypertension, metabolic diseases, cardiovascular diseases, and gastroesophageal reflux disease (35).

Other researchers investigated the influence some antihypertensive drugs have on RS debut.
Spoendlin J et al ruled out the increase of RS incidence after calcium channel blockers use and in addition sustained that beta-blockers might have protective effect against incident RS. Neither angiotensin-converting-enzyme inhibitors nor angiotensin receptor blockers have any influence on the relative RS risk. However the study does not evaluate the effect the antihypertensive drugs might have on already developed RS, but considering the results of the study, the authors estimate that calcium channel blockers do not worsen RS course while betablockers are especially benefic in erythematotelangiectatic RS treatment (36). Among the beta-blockers, atenolol and bisoprolol are the ones which seem to have protective role according to Spoendlin J et al, whereas propranolol, drug usually used off-label for erythematotelangiectatic RS did not offer any protection. As far as beta-blockers are concerned, other authors revealed that there definitely is a benefit in using either carvedilol or nadolol in RS patients, at least on small groups of patients (37-39). Spironolactone is also known to have benefic effect in RS by decreasing incidental risk for RS. The mechanism for this therapeutic end point is unknown, but it is believed that it is independent from the diuretic effect of the drug and that it is rather a consequence of the fact that by blocking the aldosteron receptor in the skin the proinflammatory effect this receptor appears to have will be inhibited (40-42).

As regarding the link RS and psychiatric diseases, Spoendlin J et al indicated that patients diagnosed with schizophrenia, independently of the psychotropic drug they were prescribed, as well as patients taking lithium on long term for any other psychiatric disease excepting schizophrenia, have a low risk for incident RS (43). The same study highlights that depression or any other affective disorder does not increase the risk for developing RS. However, RS patients can also have a psychiatric malady, especially depression, frequently as a consequence of each individual's perception on one's RS symptomatology or one's personal difficulties in the therapeutic program. Other scientific articles suggest that RS and depression, especially major depressive disease, are two entities with a reciprocal influence, psychogenic factors being important not only in the debut of RS but also in its aggravation (44).

When looking for an association between RS and immune system disorders we found that the the level of serum vitamin D was investigated in RS patients. The authors of the study reported that in the lot of RS 
patients the level of serum vitamin D was definitely higher than in the lot of healthy controls. As a direct result they concluded that vitamin $\mathrm{D}$ might have a role in RS ethiopathogenesis, especially if taking into account the role this vitamin plays in the homeostasia of the immune system (45). Also, the level of antinuclear antibodies in RS patients was broadly studied. One article from 2010 highlighted ANA in $21,1 \%$ of the patients diagnosed with RS and the authors ruled out autoimmunity involvement in RS development in the studied lot (46). In another work, as much as $53,5 \%$ of the RS patients included in the research had ANA titer higher than 1:160 (13,86\% above $1: 320,8,9 \%$ above $1: 640,6,93 \%$ above 1:1280). After a fallow-up of two years, none of the patients with a titer higher than 1:640 did not develop any symptom specific for lupus erythematosus or any other autoimmune disorder. The authors warn that ANA titers can be high in RS patients. This fact correlated with the clinical appearance and the photosensitivity characteristic to RS can make sometimes more difficult the differential diagnose with lupus erythematosus (47). If this was the case, it is recommended to make a cutaneous biopsy and/or to evaluate a larger array of antibodies (anti Ro/La, ADNdc, Sm). Also, it is advisable to fallow up patients with high antinuclear titers because Arbuckle MR et al. indicated in a previous article that symptoms of lupus can develop after a period of three to nine years after first detection of antibodies (48).

RS could also be linked to some cancers. The conclusion of a recent study of Li WQ et al was that there is an association between a medical personal history of RS and a higher risk of thyroid cancer and basal cell carcinoma (49).

$\mathrm{RS}$ is defined as a localized chronic dermatologic inflammatory disease. However, it was debated recently if as in other dermatologic inflammatory illnesses which had been thought previously to be limited to the skin, RS has the potential to amplify or to initiate to some extent the systemic inflammation syndrome. Studies done in this direction sustain the above mentioned hypothesis. In one research on a lot of 45 RS patients and a healthy control lot of 100 people, it was discovered that in the RS lot the GST (glutathione S-transferase) gene polymorphism is more frequently encountered. This genetic modification is known to be associated with an increased activity of reactive oxygen species (50). According to $\mathrm{Baz} \mathrm{K}$ et al. a high level of reactive oxygen species indicates that RS is a disease associated with oxidative stress. The same study indicates that the antioxidative activity is decreased in RS, independent of
HP coinfection, sustaining the hypothesis that in RS there is systemic inflammation, without the influence of an accidental coinfection (51).

\section{DISCUSSION}

There is still much debate weather RS is due to a particular immunologic background which can also trigger other inflammatory maladies or if RS by itself promotes a proinflammatory medium which could be a link in the chain of events that lead to a specific inflammatory pathology. It is possible to find the answer to this dilemma only when we will elucidate the etiopathogenic mechanisms of RS. However, no matter the answer, RS and concurrent inflammatory diseases tend to have a reciprocal influence on one another's course. This way, on one hand the papulopustular lesions of RS and even the intensity of facial erythema can be improved by treating nearby infections. On the other hand repeated outbreaks of infection can aggravate RS just as a severe episode of RS could aggravate some other simultaneous condition. Although inflammation is present even from the first stages of RS at least histologically, it is more plausible that papulo- pustular RS with its intense cutaneous inflammation could in a way interfere with one patient's comorbidities.

\section{CONCLUSION}

In conclusion, we can not classify rosacea as a systemic disorder, but it frequently associates with diseases, in a manner dependent of the skin lesions severity. As a consequence, patients suffering of severe forms of rosacea for a long time should be carefully evaluated as rosacea tends to associate to various inflammatory diseases which have a negative impact on its course.

\section{REFERENCES}

1. Epstein N, Susnow D. Acne rosacea: with particular reference to gastric secretion. Cal West Med. 1931 Aug;35(2):118-20.

2. Szlachcic A. The link between Helicobacter pylori infection and rosacea. J Eur Acad Dermatol Venereol. 2002 Jul;16(4):328-33.

3. Gravina A, Federico A, Ruocco E, Lo Schiavo A, Masarone M, Tuccillo $C$, et al. Helicobacter pylori infection but not small intestinal bacterial overgrowth may play a pathogenic role in rosacea. United European Gastroenterol J. 2015 Feb;3(1):17-24.

4. Utas S, Ozbakir 0, Turasan A, Utas C. Helicobacter pylori eradication treatment reduces the severity of rosacea. J Am Acad Dermatol. 1999 Mar; 40(3):433-5

5. Candelli M, Carloni E, Nista EC, Cazzato IA, Pignataro G, Rigante D, et al. Helicobacter pylori eradication and acne rosacea resolution: 
cause-effect or coincidence? Dig Liver Dis. 2004 Feb;36(2):163.

6. Bhattarai S, Agrawal A, Rijal A, Majhi S, Pradhan B, Dhakal SS. The study of prevalence of Helicobacter pylori in patients with acne rosacea. Kathmandu Univ Med J (KUMJ). 2012 Oct-Dec;10(40):4952.

7. El-Khalawany M, Mahmoud A, Mosbeh AS, A B D Alsalam F, Ghonaim $\mathrm{N}$, Abou-Bakr A. Role of Helicobacter pylori in common rosacea subtypes: a genotypic comparative study of Egyptian patients. J Dermatol. 2012 Dec;39(12):989-95.

8 Gedik GK, Karaduman A, Sivri B, Caner B. Has Helicobacter pylori eradication therapy any effect on severity of rosacea symptoms? J Eur Acad Dermatol Venereol. 2005 May;19(3):398-9.

9. Bamford JT, Tilden RL, Blankush JL, Gangeness DE. Effect of treatment of Helicobacter pylori infection on rosacea. Arch Dermatol. 1999 Jun; 135(6):659-63.

10. Argenziano G, Donnarumma G, lovene MR, Arnese P, Baldassarre MA, Baroni A. Incidence of anti-Helicobacter pylori and anti-CagA antibodies in rosacea patients. Int J Dermatol. 2003 Aug;42(8):601-4. Erratum in Int J Dermatol. 2004 Jan;43(1):83.

11. Szlachcic A, Sliwowski Z, Karczewska E, Bielanski W, Pytko-Polonczyk J, Konturek SJ. Helicobacter pylori and its eradication in rosacea. J Physiol Pharmacol. 1999 Dec;50(5):777-86.

12. Abram K, Silm H, Maaroos HI, Oona M. Risk factors associated with rosacea. J Eur Acad Dermatol Venereol. 2010 May;24(5):565-71. doi: 10.1111/j.1468-3083.2009.03472.x. Epub 2009 Oct 23.

13. Son SW, Kim IH, Oh CH, Kim JG. The response of Rosacea to eradication of Helicobacter pylori. Br J Dermatol. 1999 May;140(5):984-5.

14. Herr $\mathrm{H}$, You $\mathrm{CH}$. Relationship between Helicobacter pylori and rosacea: it may be a myth. J Korean Med Sci. 2000 Oct;15(5):551-4.

15. Bonamigo RR, Leite CS, Wagner M, Bakos L. Rosacea and Helicobacter pylori: interference of systemic antibiotic in the study of possible association. J Eur Acad Dermatol Venereol. 2000 Sep;14(5): 424-5.

16. Diaz C, O'Callaghan CJ, Khan A, Ilchyshyn A. Rosacea: a cutaneous marker of Helicobacter pylori infection? Results of a pilot study. Acta Derm Venereol. 2003;83(4):282-6.

17. Robledo MA, Orduz M. Hypothesis of demodicidosis rosacea flushing etiopathogenesis. Med Hypotheses. 2015 Apr;84(4):408-12.

18. Coyle WJ. Is rosacea another disorder of gut microbes? Curr Gastroenterol Rep. 2009 Aug;11(4):253-4.

19. Parodi A, Paolino S, Greco A, Drago F, Mansi C, Rebora A, et al. Small intestinal bacterial overgrowth in rosacea: clinical effectiveness of its eradication. Clin Gastroenterol Hepatol. 2008 Jul;6(7):759-64.

20. Romiti R, Jansen T, Heldwein W, Plewig G. Rosacea fulminans in a patient with Crohn's disease: a case report and review of the literature. Acta Derm Venereol. 2000 Mar-Apr;80(2):127-9.

21. Li WQ, Cho E, Khalili H, Wu S, Chan AT, Qureshi AA. Rosacea, use of tetracycline, and risk of incident inflammatory bowel disease in women. Clin Gastroenterol Hepatol. 2016 Feb;14(2):220-225.e3.

22. Kendall SN. Remission of rosacea induced by reduction of gut transit time. Clin Exp Dermatol. 2004 May;29(3):297-9.

23. Al-Balbeesi AO. Rosacea and chronic rhinosinusitis: a case-controlled study. Med Princ Pract. 2014;23(6):511-6.

24. Khan TT, Donaldson J, Hesse RJ. Facial dystonias and rosacea: is there an association? Orbit. 2014 Aug;33(4):276-9.

25. Spoendlin J, Voegel JJ, Jick SS, Meier CR. Migraine, triptans, and the risk of developing rosacea: a population-based study within the United Kingdom. J Am Acad Dermatol. 2013 Sep;69(3):399-406.

26. Cabete J, Serrăo V, Lestre S. Unilateral rosacea in a patient with Bell's palsy. J Dermatol. 2013 May;40(5):403-4.

27. Piccolo V, Ruocco V, Russo T, Ruotolo F, Piccolo S, Baroni A. Unilateral rosacea in patients with facial nerve palsy: a mere example of immuno-compromised district. J Dermatol. 2013 0ct;40(10):850.

28. Brinnel H, Friedel J, Caputa M, Cabanac M, Grosshans E. Rosacea: disturbed defense against brain overheating. Arch Dermatol Res. 1989; $281(1): 66-72$.

29. Kamińiska-Winciorek GM, Brzezińska-Wcisło LA. Assessment of micro-circulatory system with conventional capillaroscopy in patients with rosacea--preliminary study. Wiad Lek. 2006;59(9-10):618-22. Polish

30. Rosina P, Zamperetti MR, Giovannini A, Chieregato C, Girolomoni G. Videocapillaroscopic alterations in erythematotelangiectatic rosacea. J Am Acad Dermatol. 2006 Jan;54(1):100-4. Epub 2005 Nov 11.

31. Fonseca GP, Brenner FM, Muller Cde S, Wojcik AL. Nailfold capillaroscopy as a diagnostic and prognostic method in rosacea. An Bras Dermatol. 2011 Jan-Feb;86(1):87-90. English, Portuguese

32. Duman N1, Ersoy Evans S, Atakan N. Rosacea and cardiovascular risk factors: a case control study. J Eur Acad Dermatol Venereol. 2014 Sep;28(9):1165-9.

33. Hua TC, Chung PI, Chen YJ, Wu LC, Chen YD, Hwang CY, et al. Cardiovascular comorbidities in patients with rosacea: A nationwide case-control study from Taiwan. J Am Acad Dermatol. 2015 Aug;73(2):249-54.

34. Rainer BM, Fischer AH, Luz Felipe da Silva D, Kang S and Chien AL. Rosacea is more than skin deep: patients have higher odds of having chronic systemic diseases in a skin severity-dependent manner. Exp Dermatol. 2014 Nov;23 Suppl 2:1-13.

35. Rainer BM, Fischer AH, Luz Felipe da Silva D, Kang S, Chien AL. Rosacea is associated with chronic systemic diseases in a skin severity-dependent manner: results of a case-control study. J Am Acad Dermatol. 2015 0ct;73(4):604-8.

36. Spoendlin J, Voegel JJ, Jick SS, Meier CR. Antihypertensive drugs and the risk of incident rosacea. Br J Dermatol. 2014 Jul;171(1):130-6.

37. Hsu CC, Lee JY. Pronounced facial flushing and persistent erythema of rosacea effectively treated by carvedilol, a nonselective betaadrenergic blocker. J Am Acad Dermatol. 2012 Sep;67(3):491-3.

38. Hsu CC, Lee JY. Carvedilol for the treatment of refractory facial flushing and persistent erythema of rosacea. Arch Dermatol. 2011 Nov;147(11):1258-60.

39. Wilkin JK. Effect of nadolol on flushing reactions in rosacea. J Am Acad Dermatol. 1989 Feb;20(2 Pt 1):202-5.

40. Spoendlin J, Voegel JJ, Jick SS, Meier CR. Spironolactone may reduce the risk of incident rosacea. J Invest Dermatol. 2013 Oct;133(10): 2480-3.

41. Chantong B, Kratschmar DV, Nashev LG, Balazs Z, Odermatt A. Mineralo-corticoid and glucocorticoid receptors differentially regulate NF-kappaB activity and pro-inflammatory cytokine production in murine BV-2 microglial cells. J Neuroinflammation. 2012 Nov 28;9: 260.

42. Odermatt A, Kratschmar DV. Tissue-specific modulation of mineralocorticoid receptor function by 11beta-hydroxysteroid dehydrogenases: an overview. Mol Cell Endocrinol. 2012 Mar 24;350(2):168-86.

43. Spoendlin J, Bichsel F, Voegel JJ, Jick SS, Meier CR. The association between psychiatric diseases, psychotropic drugs and the risk of incident rosacea. Br J Dermatol. 2014 Apr;170(4):878-83.

44. Gupta MA, Gupta AK, Chen SJ, Johnson AM. Comorbidity of rosacea and depression: an analysis of the National Ambulatory Medical Care Survey and National Hospital Ambulatory Care Survey--Outpatient Department data collected by the U.S. National Center for Health Statistics from 1995 to 2002. Br J Dermatol. 2005 Dec;153(6):117681.

45. Ekiz O, Balta I, Sen BB, Dikilitaş MC, Ozuğuz P, Rifaioğlu EN. Vitamin D status in patients with rosacea. Cutan Ocul Toxicol. 2014 Mar;33(1):60-2. 
46. Lazaridou E, Apalla Z, Sotiraki S, Ziakas NG, Fotiadou C, Ioannides D. Clinical and laboratory study of rosacea in northern Greece. J Eur Acad Dermatol Venereol. 2010 Apr;24(4):410-4.

47. Woźniacka A, Salamon M, McCauliffe D, Sysa-Jędrzejowska A. Antinuclear antibodies in rosacea patients. Postepy Dermatol Alergol. 2013 Feb;30(1):1-5.

48. Arbuckle MR, McClain MT, Rubertone MV, Scofield RH, Dennis GJ, James JA, et al. Development of autoantibodies before the clinical onset of systemic lupus erythematosus. N Engl J Med. 2003 Oct 16;349(16):1526-33.
49. Li WQ, Zhang M, Danby FW, Han J, Qureshi AA. Personal history of rosacea and risk of incident cancer among women in the US. $\mathrm{Br} J$ Cancer. 2015 Jul 28;113(3):520-3.

50. Yazici AC, Tamer L, Ikizoglu G, Kaya TI, Api H, Yildirim H, et al. GSTM1 and GST1 null genotypes as possible heritable factors of rosacea. Photodermatol Photoimmunol Photomed. 2006 Aug;22(4):208-10.

51. Baz K, Cimen MY, Kokturk A, Aslan G, Ikizoglu G, Demirseren DD, et al. Plasma reactive oxygen species activity and antioxidant potential levels in rosacea patients: correlation with seropositivity to Helicobacter pylori. Int J Dermatol. 2004 Jul;43(7):494-7. 